\title{
Numerical Study of a 10 GHz Optical Flip-Flop Based on a Short Asymmetric DFB Laser
}

\author{
A. Abbasi, G. Roelkens, G. Morthier \\ Department of Information Technology, Ghent University - IMEC, Sint-Pietersnieuwstraat 41, B-9000 Gent, Belgium \\ Emails:aamin@intec.ugent.be
}

\begin{abstract}
We report on a numerical optimization of an all-optical flip-flop based on an asymmetric DFB laser. For the optimized design the repetition rate can increase up to $10 \mathrm{GHz}$ and the switching time can reduce to 5 ps.

OCIS codes: (230.1150) All-optical devices; (060.4510) Optical communications;
\end{abstract}

\section{Introduction}

All-optical signal-processing devices are expected to be essential parts of future optical-data networks [1]. All optical flip-flops are important building blocks for packet optical switching owing to their ability to store header information. They are typically based on bistable optical devices, in which switching between the two stable states happens by set and reset optical short pulses [2-5].

Our approach for the AOFF switching is based on a $\lambda / 4$-shifted DFB laser [2], which becomes bistable by injecting an external beam, with a wavelength outside the DFB laser stop-band. The bistability is due to non-linear effects having their origins in the carrier distribution (i.e. longitudinal spatial hole burning (LSHB)). In this paper we present an asymmetric DFB laser, which can switch much faster than a conventional symmetric one. In addition, our numerical results show the effect of laser length and confinement factor on the performance of the optimized AOFFs. The asymmetry is expressed by $r_{\kappa}$, which is defined as the ratio of the coupling coefficient on the right hand side of the $\lambda / 4$ phase shift to the coupling coefficient on the left hand side of it. From a fabrication point of view, the asymmetry can be easily realized by changing the duty cycle of the grating slightly.

\section{Switching principle}

As can be seen in Figure 1-a, decreasing the asymmetry coefficient while the laser is on causes strong LSHB. Since carrier density uniformity is a crucial factor to have stable lasing for a DFB laser, as we increase non-uniformity the laser becomes more vulnerable to the reset pulse and can be switched off faster. The asymmetric coefficient cannot increase more than 0.8 in this case, because the laser will not be single mode anymore (Figure 1-b). Both reset and set pulses have a duration of $100 \mathrm{ps}$ and a power of $20 \mathrm{~mW}$ in figure 1-b. Since the coupling coefficient on the right hand side is lower than on the left hand side, the output power of the asymmetric laser from the front facet (rhs facet) will be higher than for a symmetric laser which is another advantage for our design.
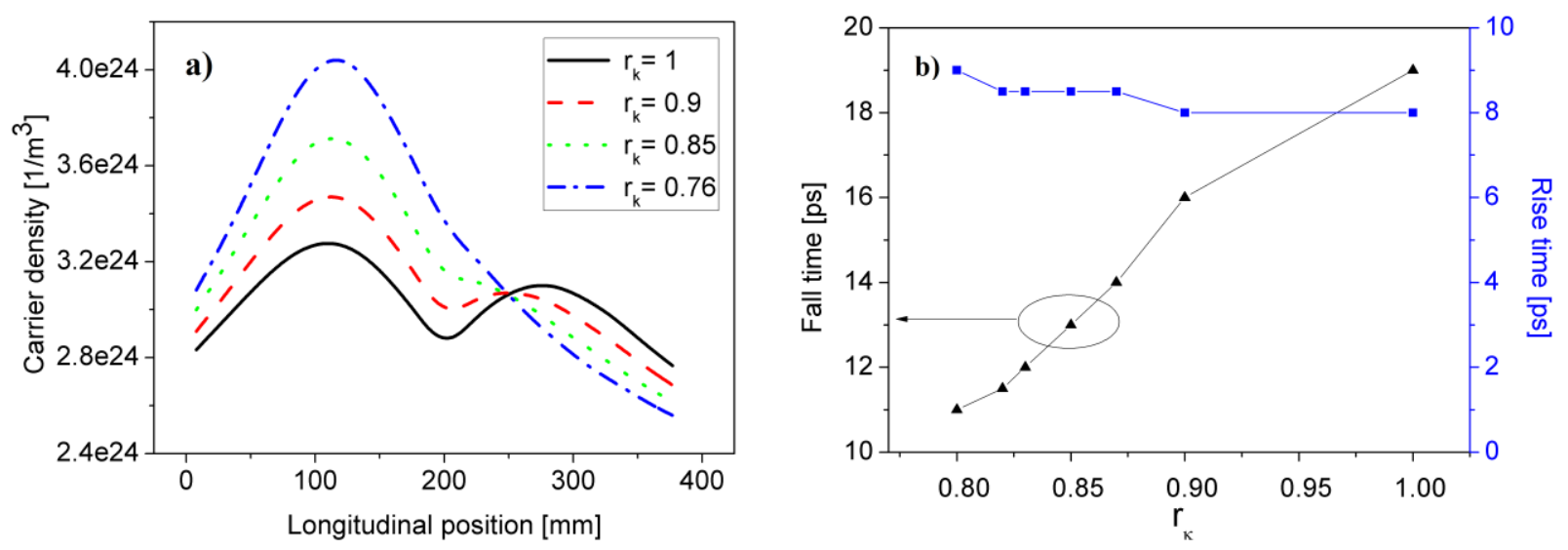

Fig. 1. The longitudinal carrier density distribution in the on-state (left) and the switching time (right) versus the asymmetry coefficient. $\mathrm{L}=400$ $\mu \mathrm{m}$, the confinement factor is $\Gamma=0.13$ and $\mathrm{I}=240 \mathrm{~mA}$.

In order to stabilize the laser operation after each switching, photons and carriers will interact within the active region of the laser. For instance, when the laser switches on by applying a set pulse, it will experience a delay time, 
a rise time and a relaxation oscillation transition. These processes happen inside the laser cavity, thus the length of the laser cavity will have a dominant influence on its switching behavior. We have also investigated the effect of the confinement factor of the QWs on the laser dynamics as well. The influence of other parameters on the switching dynamics has been investigated in ref [5]. It was shown that an increase in the differential gain led to a shorter rise time while the fall time is independent of it. Parameters like the non-linear gain coefficient and the recombination rates have very little influence.

In figure 2-a, the fall time and rise time for a laser with a length of $150 \mu \mathrm{m}$ are compared with those for a laser with a length of $400 \mu \mathrm{m}$. To keep the normalized coupling coefficient close to 1, the coupling coefficients for the lengths of $400 \mu \mathrm{m}$ and $150 \mu \mathrm{m}$ are $3000 \mathrm{~m}^{-1}$ and $6800 \mathrm{~m}^{-1}$ respectively. $\mathrm{r}_{\mathrm{\kappa}}$ equals 0.85 in both cases. The set and reset pulse widths are $25 \mathrm{ps}$ and the switching is possible even with $125 \mathrm{fJ}$ pulse energy. Since our AOFF has its origin in carrier-photon interaction, a higher confinement in the active region leads to a better performance. We have studied this effect by considering three values $7 \%, 13 \%$ and $20 \%$ for the confinement factor. To be sure that our simulated values are physically achievable for fabrication, we have chosen $13 \%$ for the optimized design. This DFB based AOFF has the ability to increase the switching speed up to $10 \mathrm{GHz}$ with a switching time of just $5 \mathrm{ps}$. The waveform of the switching with random events is plotted in figure 2-b.
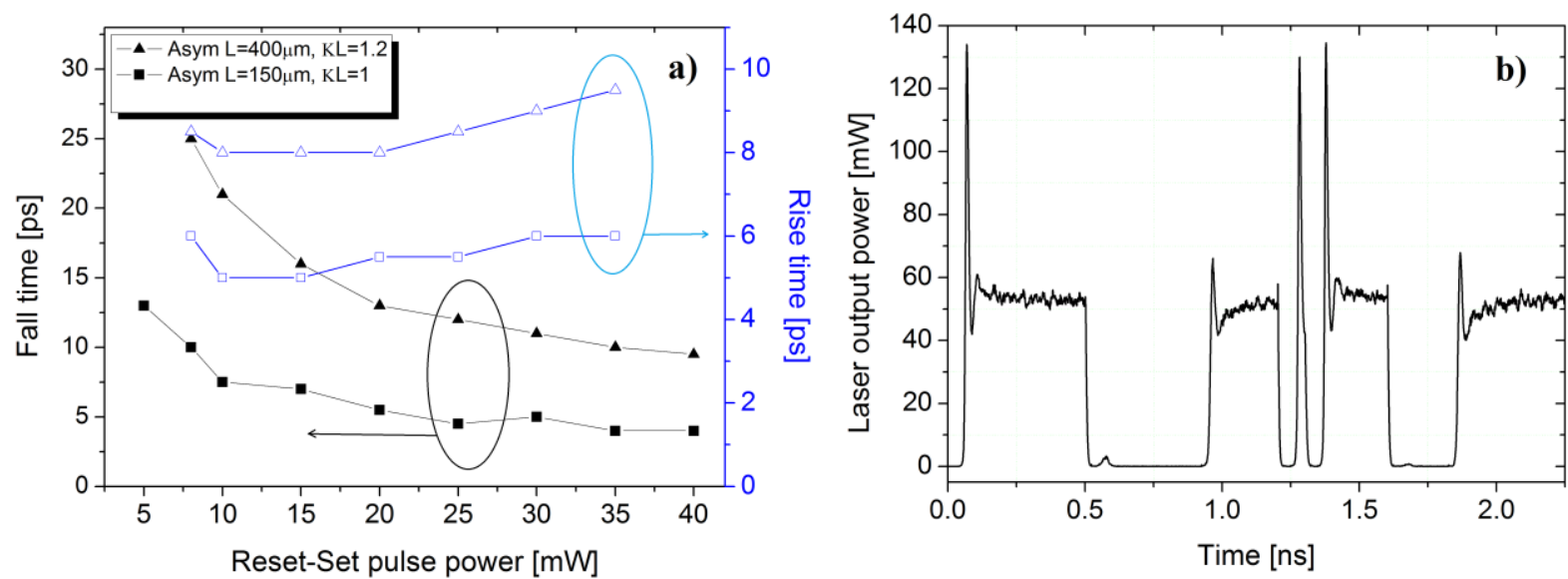

Fig. 2. The fall and rise times (left) for a $D F B$ laser with length $L=400 \mu \mathrm{m}, \kappa \mathrm{L}=1.1$ and $\mathrm{L}=150 \mu \mathrm{m}, \kappa \mathrm{L}=0.95$. The optimized Laser output waveform with random switching events (right). The asymmetry coefficient $\left(\mathrm{r}_{\mathrm{k}}\right)$ is 0.85 , the drive current is $240 \mathrm{~mA}$ and the confinement factor is 0.13 for both structures.

\section{Conclusion}

We have investigated the DFB-based AOFF optimization by taking into account the length and the asymmetry coefficient. The new design shows improvement in the maximum repetition rate up to $10 \mathrm{GHz}$, featuring very short switching times of just 5 ps.

\section{References}

[1] Agrawal, G.P, Lightwave technology: components and devices (Wiley, 2004).

[2] K. Huybrechts, G. Morthier, and R. Baets, “Fast all-optical flip-flop based on a single distributed feedback laser diode,” Opt. Express 16(15), $11405-11410$ (2008).

[3] Liu, L. et al. “An ultra-small, low-power, all-optical flip-flop memory on a silicon chip”. Nature Photon. 4, 182-187 (2010).

[4] T. Katayama, T. Kitazawa and H. Kawaguchi, "All-optical flip-flop operation using $1.55 \mu \mathrm{m}$ polarization bistable VCSELs", Conference on Lasers and Electro-Optics/Quantum Electronics and Laser Science Conference, paper CME5 (2008).

[5] K. Huybrechts, A. Ali, T. Tanemura, Y. Nakano, and G. Morthier, "Numerical and experimental study of the switching times and energies of DFB-laser based All-optical flip-flops", presented at the International Conference on Photonics in Switching, Pisa, Italy, 15-19 Sept. 2009. 